\title{
Particle production as a function of the underlying event in pp collisions simulated with PYTHIA 8
}

\author{
Gyula Bencédi, ${ }^{a, *}$ Antonio Ortiz ${ }^{a}$ and Antonio $\mathbf{P a z}^{b}$ \\ ${ }^{a}$ Instituto de Ciencias Nucleares, Universidad Nacional Autónoma de México, \\ Apartado Postal 70-543, México Distrito Federal 04510, México \\ ${ }^{b}$ Facultad de Ciencias Físico Matemáticas, Universidad Autónoma de Nuevo León, \\ Ciudad Universitaria, San Nicolas de los Garza, Nuevo León 66450, México
}

E-mail: gyula.bencedi@cern.ch

In this work we present the production of charged particles associated with high- $p_{\mathrm{T}}$ trigger particles $\left(8<p_{\mathrm{T}}^{\text {trig. }}<15 \mathrm{GeV} / c\right)$ at mid-pseudorapidity in proton-proton collisions at $\sqrt{s}=5.02 \mathrm{TeV}$ simulated with the PYTHIA 8 Monte Carlo model. The study is performed as a function of the relative transverse activity classifier, $R_{\mathrm{T}}$, which is the relative charged-particle multiplicity in the transverse region $(\pi / 3<|\Delta \phi|<2 \pi / 3)$ of the di-hadron correlations, and it is sensitive to the Multi-Parton Interactions. The evolution of the yield of associated particles on both the toward and the away regions with $3 \leq p_{\mathrm{T}}^{\text {assoc. }}<8 \mathrm{GeV} / c$ as a function of $R_{\mathrm{T}}$ is investigated. We propose a strategy which allows for the modelling and subtraction of the Underlying Event (UE) contribution from the toward and the away regions in challenging environments like those characterised by large $R_{\mathrm{T}}$. We found that the signal in the away region becomes broader with increasing $R_{\mathrm{T}}$. Contrarily, the yield increases with $R_{\mathrm{T}}$ in the toward region. This effect is reminiscent of that seen in heavy-ion collisions, where an enhancement of the yield in the toward region for $0-5 \%$ central $\mathrm{Pb}-\mathrm{Pb}$ collisions at $\sqrt{s}_{\mathrm{NN}}=2.76 \mathrm{TeV}$ was reported. To further understand the role of the UE and additional jet activity, the transverse region is divided into two one-sided sectors, "trans-max" and "trans-min" selected in each event according to which region has larger or smaller charged particle multiplicity. Based on this selection criterion, the observables are studied as a function of $R_{\mathrm{T}}^{\max }$ and $R_{\mathrm{T}}^{\min }$, respectively. The presented results have been published in Refs. [1,2].

The Ninth Annual Conference on Large Hadron Collider Physics - LHCP2021

7-12 June 2021

Online

\footnotetext{
${ }^{*}$ Speaker
} 


\section{Introduction}

One of the most important discoveries at the LHC is the presence of collectivity [3] in highmultiplicity proton-proton (pp) collisions which is originally present in heavy-ion collisions (AA) where the effect of jet quenching is observed [4]. To date no jet quenching is found in small collision systems, however several attempts have already been made to unveil it in high-multiplicity pp collisions, see e.g. Ref. [5]. To extract jet-quenching signal from pp data, one has to deal with the selection bias which is the main technical issue in the analysis. The high-activity event selection results a sample which is naturally biased toward hard processes (including jets). Following the definition by the CDF Collaboration, the direction of the trigger particle is used to define regions in the azimuthal plane that have different sensitivity to the underlying event (UE). The toward and the away regions are dominated by string fragments originating from the hardest partonic process of the event, and are expected to be nearly insensitive to the softer UE. In contrast, the trans. region is the most sensitive to UE, but it has contributions from initial- and final-state radiation (ISR and FSR) that accompanies the hard scattering. This UE region will be used to isolate events with exceptionally large or small activity with respect to the event-averaged mean.

In this work we explore a quantity called $I_{\mathrm{pp}}$ which is motivated by the $I_{\mathrm{AA}}$ [6-8] commonly used in AA collisions to study jet quenching effects. Also, we further investigate the UE-dominated trans. region considering a refinement to distinguish between the more and the less active sides of the trans. region on a per-event basis [9-11]. The purpose of using this geometrical selection criterion is to suppress hard ISR/FSR thus increasing the sensitivity of the trans. region to MPI component of the UE. Based on the CDF approach [12], the so-called trans-min and trans-max regions are introduced. The trans-min region is insensitive to wide angle emissions from the hard process, while the trans-max region receives contribution from hard ISR and/or FSR. We study the structures of the $\Delta \phi$ distribution for the trans-max and trans-min regions. The presented results are also discussed in the context of recent ALICE preliminary results [13, 14].

\section{Classification of event activity using the underlying event}

We simulated inelastic pp collisions at $\sqrt{s}=5.02 \mathrm{TeV}$ and only primary charged particles [15] with $p_{\mathrm{T}}>0.5 \mathrm{GeV} / c$ and pseudorapidity $|\eta|<0.8$ are considered in the analysis. We use the Relative Transverse Activity Classifier, $R_{\mathrm{T}}$, which has been recently introduced $[16,17]$ and used on LHC data $[18,19] . R_{\mathrm{T}}$ uses the conventional definition of the transverse region, which was adopted in the UE analysis originally introduced by the CDF collaboration [20,21]. We classify events with a trigger particle in the range $8 \leq p_{\mathrm{T}}^{\text {trig. }}<15 \mathrm{GeV} / c$ based on their per-event activity in the trans. region with respect to the mean: $R_{\mathrm{T}}=N_{\mathrm{ch}}^{\text {trans. }} /\left\langle N_{\mathrm{ch}}^{\text {trans. }}\right\rangle$. Also, we define two regions that are characterized in terms of their relative charged-particle multiplicities, $N_{\mathrm{ch}}^{\text {trans. max }}$ and $N_{\mathrm{ch}}^{\text {trans. min }}$, termed trans-max and trans-min. Trans-max (trans-min) refers to the trans, region containing the largest (smallest) number of charged particles. Using $N_{\mathrm{ch}}^{\text {trans. min }}$ and $N_{\mathrm{ch}}^{\text {trans. max }}$, instead of $N_{\mathrm{ch}}^{\text {trans. }}$, we also can define the quantities $R_{\mathrm{T}}^{\min }$ and $R_{\mathrm{T}}^{\max }$, respectively. For $R_{\mathrm{T}}, R_{\mathrm{T}}^{\max } \approx 8$ and $R_{\mathrm{T}}^{\min } \approx 8$, the inclusive multiplicity reach is about six- and four times the mean multiplicity. The average $N_{\text {mpi }}$ increases with $R_{\mathrm{T}}^{\min }$ as opposed to what is observed for $R_{\mathrm{T}}^{\max }$. Results of the correlation between 

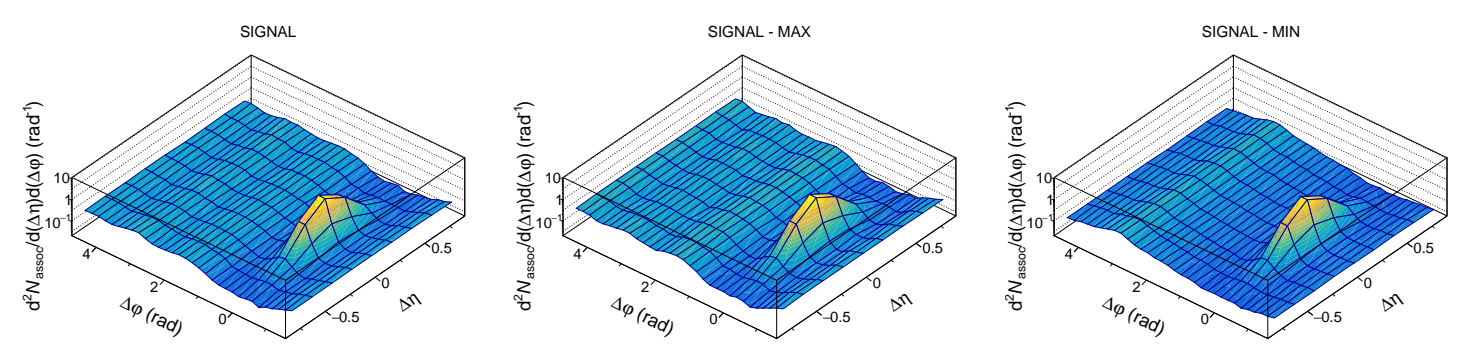

Figure 1: Charged particle yield as a function of $\Delta \eta-\Delta \phi$ for $R_{\mathrm{T}} \geq 3.5$ and $4 \leq p_{\mathrm{T}}^{\text {assoc. }}<6 \mathrm{GeV} / c$ simulated with PYTHIA 8 in pp collisions at $\sqrt{s}=5.02 \mathrm{TeV}$. Second and third columns represent the cases of trans-max and trans-min corresponding to high and low activities in the transverse region.

the average $N_{\text {mpi }}$ and $R_{\mathrm{T}}$ showed that events selected with $R_{\mathrm{T}}>2$ but with similar $N_{\text {mpi }}$ include additional jets to enhance the activity in the trans. region which in turn causes a selection bias.

\section{Extraction of jet-like signals}

Figure 1 shows the charged particle yield as a function of $\Delta \eta-\Delta \phi$ for $R_{\mathrm{T}} \geq 3.5$. A structure elongated in $\Delta \eta$ is present in the trans. region which is a consequence of the event selection. Therefore, its contribution to the toward and the away regions has to be removed. For this purpose mixed events are used to model the uncorrelated contribution as well as the acceptance effect. The signal is extracted after removing the mixed event distribution from the same event distribution. The $\Delta \eta-\Delta \phi$ distributions are flat in $\Delta \eta$, therefore, the underlying event contribution to the toward and away regions is subtracted with the zero yield at minimum assumption [22].

\section{Results}

We calculated the $I_{\mathrm{pp}}$ quantity, which is the ratio of yields from different $R_{\mathrm{T}}$ classes to the $R_{\mathrm{T}}$-integrated one. In the absence of either selection bias or jet quenching, $I_{\mathrm{pp}}$ is expected to be consistent with unity. The influence on the jet-like signals of the remaining structures are reduced by integrating the $\Delta \phi$ distribution within the interval $|\Delta \phi|<0.8(|\Delta \phi-\pi|<0.8)$ for the toward

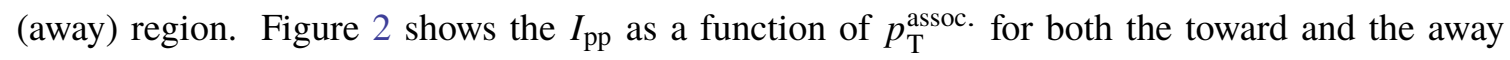

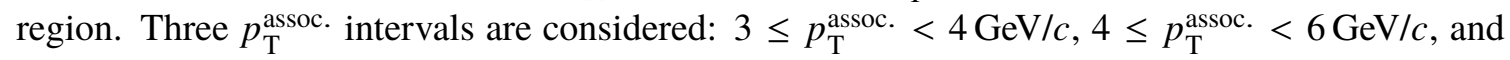
$6 \leq p_{\mathrm{T}}^{\text {assoc. }}<8 \mathrm{GeV} / c$. Results with and without underlying event subtraction are also presented. We observe that the $I_{\mathrm{pp}}$ quantity is independent of $R_{\mathrm{T}}$ in simulations which do not include ISR and FSR. With physical settings (including ISR/FSR) the yield in the toward region increases with $R_{\mathrm{T}}$; this effect is reminiscent of the $\mathrm{Pb}-\mathrm{Pb}$ data from the ALICE and CMS experiments, although in the PYTHIA 8 model it is driven by a bias, and has to be taken into account in any analysis which use $R_{\mathrm{T}}$ as an event classifier. The bias originates from hard Bremsstrahlung gluons that can produce an apparent modification of the jet-like yield in events with extremely large underlying event. In contrast, the $I_{\mathrm{pp}}$ in the away region is consistent with unity, and independent of $R_{\mathrm{T}}$ and $p_{\mathrm{T}}^{\text {assoc. }}$. This suggests that PYTHIA 8 can partially mimic jet-quenching effects.

We modified of the original $R_{\mathrm{T}}$ definition: split the transverse region into the trans-min and trans-max regions in order to control the sensitivity to hard processes. Using $R_{\mathrm{T}}^{\min }$ instead of $R_{\mathrm{T}}$ or 


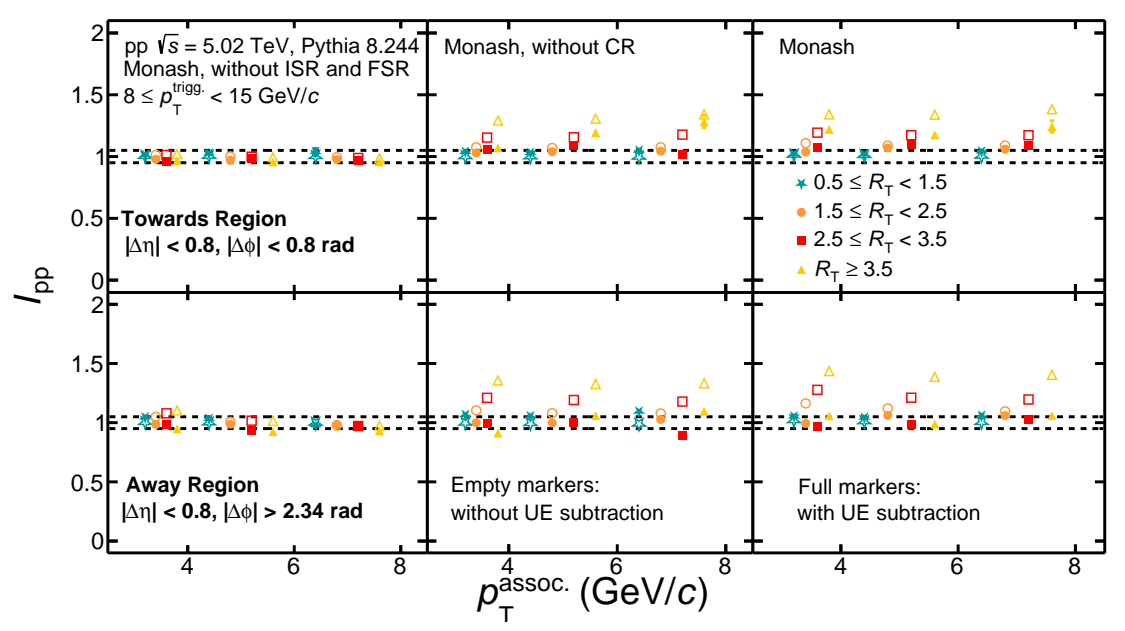

Figure 2: $I_{\mathrm{pp}}$, the ratio of yield from different $R_{\mathrm{T}}$ classes and from the $R_{\mathrm{T}}$-integrated class as a function of $R_{\mathrm{T}}$ for the toward (top) and away region (bottom). Dashed lines indicate $\pm 5 \%$ deviation from unity.

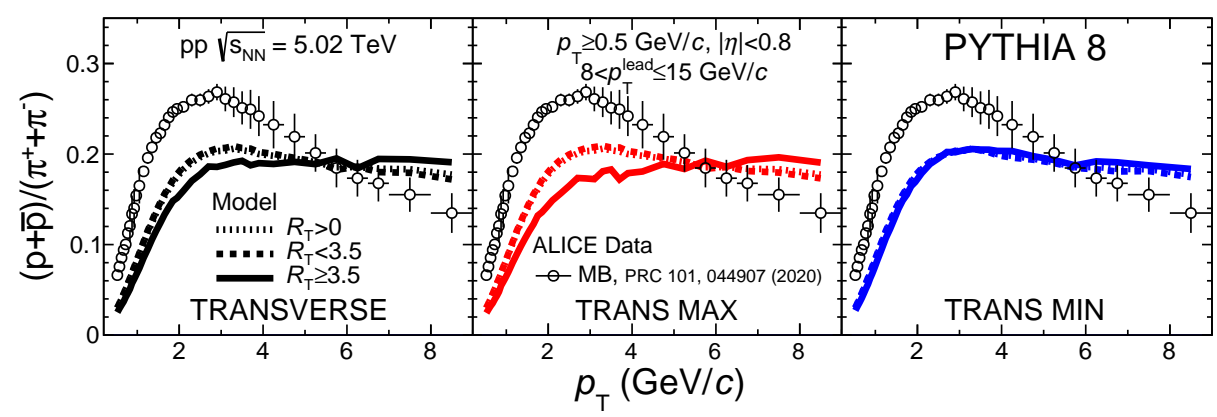

Figure 3: Proton-to-pion ratio as a function of $p_{\mathrm{T}}$ for the transverse (left panel), trans-max (middle panel), and trans-min (right panel) regions for pp collisions at $\sqrt{s}=5.02 \mathrm{TeV}$.

$R_{\mathrm{T}}^{\max }$, we do not observe a remarkable evolution of both the toward and away regions with increasing $R_{\mathrm{T}}^{\min }$, moreover, the remaining signal in the transverse region is much smaller and roughly $R_{\mathrm{T}}^{\min }$ independent. The $p_{\mathrm{T}}$ dependent $\mathrm{p} / \pi$ ratios shown in Fig. 3 for various $R_{\mathrm{T}}$ event classes exhibit a depletion at $p_{\mathrm{T}} \approx 2-3 \mathrm{GeV} / c$ with increasing $R_{\mathrm{T}}$ which is consistent with the presence of jets in the trans. region. As reported earlier both in MC [23] and data [24], the ratio is smaller in the jet region than in the UE region. The $R_{\mathrm{T}}^{\max }$-dependent ratios receive a large amount of particles from hard Bremsstrahlung gluons produced in ISR/FSR radiation.

The middle panel of Fig. 3 reports the high-activity trans. region which receives a large amount of particles from hard gluon-radiated processes, i.e. from ISR/FSR. In effect, the bump structure is smeared out; a similar effect is seen as for particle ratios inside jets [24]. In contrast, the $R_{\mathrm{T}}^{\min }$-dependent ratios shown in the right panel of Fig. 3 exhibit an opposite behaviour: only a weak dependence on the $R_{\mathrm{T}}^{\mathrm{min}}$ event classes is seen. However, the position of its maximum is slightly shifted to higher $p_{\mathrm{T}}$ values in events with large $R_{\mathrm{T}}^{\mathrm{min}}$, which is expected in PYTHIA 8 events with large $N_{\text {mpi }}$ and color reconnection [25-27]. 


\section{Acknowledgments}

This work has been supported by CONACyT under the Grants No. A1-S-22917 and CF-2042. G. B. acknowledge the postdoctoral fellowship grant provided by CONACyT under the Grant No. A1-S-22917. The support for part of this work has been received during the pandemic by the National Research, Development and Innovation Office (NRDIO) OTKA K120660 and FK131979,20192.1.11-TET-2019-00078(HuMex) and 2019-2.1.6-NEMZKI-2019-00011 (CERN).

\section{References}

[1] G. Bencédi, A. Ortiz and S. Tripathy, J. Phys. G 48, no.1, 015007 (2020)

[2] G. Bencedi, A. Ortiz and A. Paz, Phys. Rev. D 104, no.1, 016017 (2021)

[3] J. L. Nagle and W. A. Zajc, Ann. Rev. Nucl. Part. Sci. 68, 211-235 (2018)

[4] U. A. Wiedemann, doi:10.1007/978-3-642-01539-7_17 [arXiv:0908.2306 [hep-ph]].

[5] P. M. Jacobs [ALICE], Nucl. Phys. A 1005, 121924 (2021)

[6] J. Adams et al. [STAR], Phys. Rev. Lett. 97, 162301 (2006)

[7] A. Adare et al. [PHENIX], Phys. Rev. Lett. 104, 252301 (2010)

[8] K. Aamodt et al. [ALICE], Phys. Rev. Lett. 108, 092301 (2012)

[9] G. Marchesini and B. R. Webber, Phys. Rev. D 38, 3419 (1988)

[10] J. Pumplin, Phys. Rev. D 57, 5787-5792 (1998)

[11] D. Acosta et al. [CDF], Phys. Rev. D 70, 072002 (2004)

[12] R. Field, Ann. Rev. Nucl. Part. Sci. 62, 453-483 (2012)

[13] S. Tripathy [ALICE], [arXiv:2103.07218 [hep-ex]].

[14] A. Nassirpour [ALICE], J. Phys. Conf. Ser. 1602, no.1, 012007 (2020)

[15] S. Acharya et al. [ALICE], https://cds.cern.ch/record/2270008

[16] T. Martin, P. Skands and S. Farrington, Eur. Phys. J. C 76, no.5, 299 (2016)

[17] A. Ortiz and L. Valencia Palomo, Phys. Rev. D 96, no.11, 114019 (2017)

[18] S. Acharya et al. [ALICE], JHEP 04, 192 (2020)

[19] V. Zaccolo [ALICE], Springer Proc. Phys. 250, 245-248 (2020)

[20] R. Field [CDF], Int. J. Mod. Phys. A 16S1A, 250-254 (2001) FERMILAB-CONF-00-289-E.

[21] T. A. Aaltonen et al. [CDF], Phys. Rev. D 92, no.9, 092009 (2015)

[22] N. N. Ajitanand, et al., Phys. Rev. C 72, 011902 (2005)

[23] A. Ortiz, G. Paić and E. Cuautle, Nucl. Phys. A 941, 78-86 (2015)

[24] A. Zimmermann [ALICE], J. Phys. Conf. Ser. 805, no.1, 012009 (2017)

[25] A. Ortiz et al., Phys. Rev. Lett. 111, no.4, 042001 (2013)

[26] A. Ortiz, et al., Phys. Rev. D 102, no.7, 076014 (2020)

[27] A. Ortiz, et al., J. Phys. G: Nucl. Part. Phys. 48085014 (2021) 